\title{
Reformas del sector de la salud y participación social
}

\author{
Carmen Celedón ${ }^{1}$ y Marcela Noé2
}

RESUMEN Tras una breve revisión de las diversas formas de entender la participación ciudadana en materia de salud, este artículo plantea la necesidad de que existan mecanismos de control social de cada una de las funciones que debe cumplir el sistema de salud: definición de politicas y re gulación, financiamiento, aseguramiento, producción de servicios y evaluación de la eficacia y eficiencia del sistema. En general, en los países de América Latina y el Caribe la formulación de políticas en el sector de la salud continúa realizándose de forma centralizada, con escasa par ticipación de las personas y baja adecuación a la realidad local. Aunque los procesos de reforma en la Región están avanzando en la definición o perfeccionamiento de la función de financia miento para conciliar mejor los objetivos de equidad, eficiencia y libertad de elección, el desarrollo de instrumentos que permitan ejercer control ciudadano sobre las funciones de financia miento y de aseguramiento del sistema es aún muy precario. Pese a los avances realizados, también se está lejos de contar con instrumentos apropiados para que la ciudadanía ejerza un control efectivo sobre los prestadores de servicios de salud. Entre los principales obstáculos para el desarrollo de la participación ciudadana en el sector destacan: la desigualdad en la dis tribución de los ingresos y de otras formas de poder que caracteriza a los países de la Región, el carácter incipiente del reconocimiento de los derechos de las personas frente a los servicios públicos, la escasa información acerca de esos derechos y la ausencia de mecanismos que permitan hacerlos efectivos, la precariedad con que los mecanismos de control existentes recono cen la diversidad social y recogen la opinión de las minorías o de los sectores más marginados, y el débil desarrollo de las organizaciones sociales.

En una sociedad democrática la ciudadanía consiste en el derecho de las personas a definir las reglas del juego, los derechos y las obligaciones y a contar con mecanismos que garanticen que estos derechos se hagan efectivos. La experiencia histórica revela que la participación ciudadana en materia de salud ha sido entendida de diversas formas:

1 Universidad Católica de Chile, Chile. Dirección postal: c/Fidel Oteiza 1956, Of. 21, Providencia, Santiago, Chile. Correo electrónico: cceledonc@ hotmail.com

2 Universidad Católica de Chile, Santiago, Chile. Correo electrónico: mnoe@entelchile.net
A. La participación como beneficiario, que ha sido la forma más rudimentaria de interpretar la participación por parte de los organismos gubernamentales responsables de la salud en los distintos países, considera a las personas como simples receptoras o beneficiarias de la atención de salud. Aun en estos días es difícil romper la tradicional relación vertical propia del sector de la salud, que les asigna escasos espacios de participación a las personas. Esta ha sido la visión predominante en las organizaciones creadas bajo el concepto de gratuidad de la salud en aquellos países donde el sector público de la salud ha tenido un papel y desarrollo preponderantes.

B. La participación como contribución a la promoción de la salud. En el sector de la salud es frecuente definir la participación como el apoyo voluntario de la población a determinados programas y proyectos de desarrollo social establecidos por los servicios de salud o las autoridades locales. Está claro que la contribución de la comunidad es fundamental para lograr el éxito de estos programas. Sin embargo, bajo este concepto de participación se mantiene una relación de verticalidad entre el agente 
público y las personas, que son vistas como un instrumento o recurso que facilita la acción del Estado.

C. La participación comunitaria, que en general promueven los organismos multilaterales, interpreta la participación como la organización de la comunidad en comités o grupos de apoyo a los programas y proyectos de desarrollo social. El objetivo de la organización es, además de la obtención de recursos de la comunidad, transmitir conocimientos y capacitar a la población para que se transforme en un agente activo de su desarrollo. Esta visión más completa de la participación asigna mayores espacios de acción a la ciudadanía. Sin embargo, en una sociedad en modernización, con una creciente heterogeneidad de intereses, visiones y modos de vida de las personas, es difícil identificar a la "comunidad" como una entidad que actúe ante el sector de la salud como interlocutor único o en representación de esta diversidad social. En este contexto de heterogeneidad social, los instrumentos de participación colectivos tradicionales son cada vez menos eficaces para recoger las demandas de los ciudadanos y su limitación es aún mayor en países que presentan un escaso desarrollo de sus redes sociales, como ocurre en la mayor parte de los países de la Región de las Américas (1).

D. Una visión más moderna de participación en la salud reconoce a las personas como usuarios y a la vez como ciudadanos ante los cuales el sistema de salud debe rendir cuentas de la calidad y oportunidad del servicio, así como del uso eficiente de los recursos. Surge entonces la demanda de una mayor responsabilidad del sistema frente a la ciudadanía y la necesidad de implementar distintos mecanismos de participación, ya sean individuales o colectivos, que permitan a las personas ejercer control sobre sus derechos de protección de la salud (2).

Este artículo aborda el tema de la participación como mecanismo de con- trol social, entendiendo como tal la capacidad de las personas de hacer valer sus derechos a la protección de la salud ante el sistema de salud. En él se plantea la necesidad de que existan mecanismos de control social en cada una de las funciones que debe realizar el sistema: definición de políticas y regulación (función normativa), financiamiento, aseguramiento o compra de bienes y servicios de salud y producción de servicios de salud (3). A ellas puede agregarse la función de evaluación, que consiste en velar por la eficacia (capacidad de lograr los objetivos propuestos) y la eficiencia (obtenerlos al mínimo costo) del sistema. La existencia de mecanismos de evaluación del sistema de salud es responsabilidad del Estado y el ejercicio de esta función debe ser compartido por el Estado y los ciudadanos. Finalmente, se realizan algunas reflexiones acerca de la medida en que la participación y el control ciudadano se ven potenciados por los procesos de reforma del sector que se están llevando a cabo en la Región.

\section{PARTICIPACIÓN Y CONTROL SOCIAL EN LA DEFINICIÓN DE POLÍTICAS Y LA REGULACIÓN}

Hacer valer los derechos de las personas requiere la existencia de ciertos mecanismos de control por parte de la ciudadanía. Estos pueden ser instrumentos de mercado, legales o de participación, tanto individual como colectiva. Los distintos instrumentos de control social requieren cierto contexto para ser eficaces y eficientes, favorecer la equidad y promover la formación de capital social. Se requieren también ciertas condiciones para que estos instrumentos sean sostenibles. Por ejemplo, los mecanismos de mercado funcionan mejor en contextos en los que existe mejor información y una distribución más igualitaria de los ingresos; los mecanismos legales son más eficaces y equitativos cuando existe un estado de derecho más desarrollado, y los mecanismos de participación funcionan mejor en sociedades en las que existe una mayor cohesión social y confianza en las instituciones (2).
El Estado es responsable de ejercer la función normativa del sistema, la cual básicamente consiste en la definición de políticas y la regulación. Sin embargo, el que esta función sea responsabilidad del Estado no debe confundirse con que deba ser ejercida autoritariamente, desde la burocracia técnica y sin la participación de la ciudadanía. Al contrario, existen claras ventajas al incorporar en su definición a los actores afectados por las políticas públicas, y no solo como expresión real de la democracia política, sino también como elemento creador de condiciones que favorecen la efectividad y la sostenibilidad técnica y política. En efecto, la incorporación de los actores afectados por las políticas públicas permite responder mejor a las necesidades de la ciudadanía, posibilita una mejor definición técnica de las normas y facilita su aplicación. Por otra parte, un proceso participativo de definición de políticas habilita a las personas como ciudadanos conscientes de sus derechos, facilita que ejerzan posteriormente un efectivo control social sobre el sistema, e incrementa el capital social, lo cual favorece su sostenibilidad $(4,5)$.

En general, en los países de América Latina y el Caribe la formulación de políticas en el sector de la salud continúa realizándose de forma centralizada, con escasa participación de las personas y baja adecuación a la realidad local. En Chile, por ejemplo, si bien existe una Ley Orgánica Constitucional de Gestión de Municipalidades que establece la obligatoriedad de contar con un Plan de Salud Comunal generado tras un diagnóstico con la participación de los ciudadanos, su implementación es aún precaria en la mayor parte de las comunas del país. En este proceso, liderado por el sector público de la salud, participan normalmente organizaciones sociales tradicionales o de voluntariado que no expresan la diversidad social. Los grupos o redes sociales emergentes, propios de una sociedad en modernización y más individualista, se reúnen en torno a problemáticas comunes, tales como temas medioambientales, culturales o de género. Estas redes se caracterizan por su gran 
dinamismo en la vida social, pero son frecuentemente de carácter informal y esporádico, por lo que no siempre son convocadas a estos procesos de diagnóstico, como tampoco lo son las empresas privadas del sector de la salud. Por otra parte, los objetivos sanitarios son priorizados en el nivel central y, si bien se han abierto espacios para la incorporación de objetivos locales, no existen instrumentos ni capacidades en este nivel para que estos derechos puedan hacerse efectivos.

El contexto de globalización, de mayor crecimiento económico y de participación del sector privado en la salud está generando ciudadanos más informados, que tienen mayores expectativas y son más exigentes frente a los servicios de salud. Sin embargo, las aspiraciones de los ciudadanos se modifican a mayor velocidad que la capacidad de respuesta del sistema de salud. El proceso de cambio de la regulación desde una normativa desarrollada bajo una lógica de Estado benefactor y prestador de servicios de salud hacia una regulación pro-competitiva y con mayor participación y protección de los ciudadanos requiere un largo período de desarrollo. En este ámbito, los países de América Latina y el Caribe presentan grandes carencias, que se han visto acentuadas con las reformas en marcha.

\section{PARTICIPACIÓN Y CONTROL SOCIAL EN LA FUNCIÓN DE FINANCIAMIENTO}

Como es sabido, la sola operación del mercado no garantiza la equidad ni la eficiencia en la función de financiamiento del sistema de salud. Por ello, la acción del Estado y la participación y control por parte de los ciudadanos son fundamentales para un eficiente desempeño de esta función.

La función del Estado en el financiamiento consiste en establecer las fuentes de recursos y las reglas de recaudación y de asignación de subsidios, así como velar por el cumplimiento de estas. Esta función se funde, a menudo, con las de regulación y aseguramiento, porque, para garantizar el acceso uni- versal a los seguros de salud, es necesario que se aplique el principio de no exclusión; es decir, que toda persona sea aceptada en el sistema, que se desarrollen mecanismos que limiten las fallas del mercado asegurador y que se defina el nivel de protección de la salud garantizado a los ciudadanos para que estos derechos puedan ser exigibles.

La combinación de los objetivos que debe perseguir el Estado (equidad, eficiencia y libertad de elección) con las limitaciones de las personas para ejercer la función de financiamiento de forma individual hace que en algunos países el modelo de financiamiento esté basado en empresas o asociaciones territoriales, como organizaciones que pueden reunir a grupos de personas que resuelven colectivamente el problema de adquirir los seguros de salud. Sin embargo, un problema común al ejercicio de la función de financiamiento por parte del Estado, o por cualquier otra entidad pública o privada, es el problema del agente principal. Esto es, que el intermediario (Estado, empresas o asociaciones geográficas) efectivamente asigne el financiamiento en favor de los usuarios (ciudadanos) para asegurar un adecuado nivel de protección. Se requiere entonces que los ciudadanos puedan ejercer control social sobre estas entidades para limitar la captura por parte de las empresas de seguros, problema que se hace tanto más agudo cuanto más interesante sea la cartera que maneja el intermediario y menor sea el grado de competencia en el mercado asegurador. ${ }^{3}$

Los procesos de reforma en la Región están avanzando en la definición o perfeccionamiento de la función de financiamiento con el propósito de conciliar mejor los objetivos de equidad, eficiencia y libertad de elección. Sin embargo, el desarrollo de instrumentos que permitan ejercer control ciudadano sobre las funciones de financiamiento y de aseguramiento del sistema de salud es aún muy precario.

\footnotetext{
3 Celedón C, Oyarzo C. Hacia una garantía universal en salud. Una nueva relación entre lo público y lo privado. Documento preparado para el Foro de Desarrollo Productivo del Ministerio de Economía de Chile (1999), financiado por la Fundación Ford.
}

En el sector no se han desarrollado organizaciones de consumidores o grupos de defensa de los derechos ciudadanos, como tampoco ha ocurrido en otros sectores de la economía. Y ello porque nuestras sociedades se han caracterizado históricamente por un débil desarrollo de sus redes sociales.

\section{PARTICIPACIÓN Y CONTROL SOCIAL EN LA FUNCIÓN DE ASEGURAMIENTO O DE COMPRA DE SERVICIOS}

La función de aseguramiento consiste en la canalización de recursos hacia la compra de bienes y servicios de salud. La existencia de organismos aseguradores/compradores tiene por función promover la eficiencia de los prestadores. Un asegurador/comprador con mayor información que la que poseen los individuos, que vele por la pertinencia y calidad de las prestaciones de salud, se justifica debido a la existencia de altas asimetrías de información en el mercado de la atención sanitaria. Los individuos tienen dificultades para discernir la pertinencia de las acciones de salud y no tienen la capacidad de controlar los costos de las prestaciones. Los organismos aseguradores/compradores pueden ser públicos o privados y no tienen por qué ser únicos, lo cual permite también una mayor amplitud de elección por parte de las personas.

El papel de las asociaciones de consumidores o de afiliados al sistema de seguros de salud es fundamental en la función de articulación porque permite ejercer control social sobre los compradores estatales o privados. También la información y los mecanismos legales son claves para el control ciudadano en el ejercicio de esta función.

Como ocurre con la función de financiamiento, los procesos de reforma en la Región han puesto escaso énfasis en promover el desarrollo de mecanismos individuales o colectivos que permitan ejercer control social sobre los compradores del sistema. En algunos países las entidades aseguradoras están controladas por organismos públicos especializados (por ejemplo, la 
superintendencia del ramo) que actúan en representación de los intereses comunes de los usuarios. Sin embargo, estas entidades operan en un contexto de precariedad de recursos y deben hacer frente permanentemente a fuertes presiones por parte de los grupos más organizados o los intereses corporativos del sector. Una mayor información de los ciudadanos y su incorporación al control de los seguros de salud permitiría a esas entidades un mejor cumplimiento de esta función social.

\section{PARTICIPACIÓN Y CONTROL SOCIAL EN LA PRESTACIÓN DE SERVICIOS DE SALUD}

En general, la prestación de servicios de salud en los países de América Latina y el Caribe se caracteriza por un modelo mixto y segmentado, en el cual los prestadores públicos atienden a la población de mayor riesgo y de menores ingresos. Los prestadores públicos presentan problemas de gestión y de eficiencia propios de organizaciones que operan en un contexto burocrático, con escasa competencia y falta de control efectivo por parte de la administración, a lo que se agrega, en algunos países de América Latina y el Caribe, una falta de recursos para el sector. Otro elemento que dificulta la gestión de los hospitales y consultorios públicos es el excesivo centralismo en las políticas de gestión de los recursos humanos y financieros. Por su parte, los prestadores privados operan en un contexto de mayor flexibilidad, lo que les permite adecuarse más fácilmente a las demandas de los usuarios. Sin embargo, los ciudadanos se ven enfrentados a mercados con escasa información y deficiente regulación, lo que les impide tomar decisiones racionales en cuanto a la calidad y costo de los servicios de salud.

La influencia política y el poder social que tradicionalmente han tenido los gremios de los profesionales de la salud, reforzados por la relación vertical entre el médico y el paciente, han tenido preponderancia sobre la opinión de los ciudadanos en un contexto en el que existen escasos mecanismos de control social sobre los prestadores de servicios de salud. Por ello, una parte importante de los aumentos de recursos destinados al sector de la salud que han acompañado a las reformas ha generado aumentos de los costos del sistema que no se han traducido necesariamente en mejoras equivalentes en la calidad del servicio.

Dada la insatisfacción general de los ciudadanos con el sector de la salud, en diversos países se han realizado esfuerzos orientados a recoger la opinión de los ciudadanos y a establecer un mayor control sobre los prestadores de servicios de salud. En el sector público de la salud este control se ha ejercido a través de diversos mecanismos, entre ellos la implementación de sistemas de información y reclamación, la realización de encuestas de opinión y el establecimiento de compromisos sobre cobertura, calidad y oportunidad del servicio, como son los compromisos de gestión entre el Ministerio de Salud y los servicios de salud en varios países, como Chile y Costa Rica (5). Aunque ello representa un avance en la dirección deseada, todavía estamos lejos de contar con instrumentos apropiados para que la ciudadanía ejerza un control efectivo sobre los prestadores de servicios de salud.

\section{EL APORTE DE LAS REFORMAS A LA PARTICIPACIÓN Y EL CONTROL CIUDADANO EN SALUD}

Los procesos de reforma del sector de la salud que se están llevando a cabo en América Latina y el Caribe están orientados a que el sistema de salud responda con mayor eficiencia y equidad a las necesidades de los ciudadanos. Desde esta perspectiva se está avanzando en las siguientes líneas de acción:

A. Definición de una garantía en salud. El diagnóstico de las necesidades de la población y la capacidad que tiene el sistema de garantizar su satisfacción, con calidad y oportunidad de servicio a todos los ciudadanos, permite definir dicha garantía, que es una condición esencial para que los ciudadanos puedan exigir sus derechos. Algunos países, como Argentina y Colombia, han definido esta garantía mediante el establecimiento de un plan básico o garantizado para todos los ciudadanos. Otros, como Chile, están avanzando en esta tarea mediante la definición de una carta de derechos ciudadanos, siguiendo el ejemplo de Gran Bretaña y de otros países desarrollados.

B. Separación de las funciones de finan ciamiento, articulación y prestación. Una dirección común en los procesos de reforma en la Región es la separación de estas funciones. En aquellos países, como Chile, en los que el sector público de la salud es predominante, al inicio de los procesos de reforma se contaba con una organización jerárquica y burocrática sin separación de funciones. En los últimos años se ha avanzado en la delimitación de responsabilidades, en particular en la definición de una función de financiamiento y en la separación de las funciones de compra y de prestación de servicios, con el objeto de mejorar la eficiencia del sistema (6). Este proceso, aún incipiente, incorpora instrumentos de control ciudadano sobre el sistema que todavía están en proceso de formulación (carta de derechos ciudadanos).

C. Introducción de incentivos y fomento de la competencia. Los procesos de reforma del sector están introduciendo incentivos a la eficiencia y a la capacidad de respuesta a las demandas de los ciudadanos a través de contratos internos y de una mayor competencia entre aseguradores y prestadores de servicios de salud. Estos cambios otorgan a los ciudadanos un mayor control sobre el sistema, gracias a la posibilidad de elegir la agencia aseguradora y prestadora. Este poder es mayor en los mercados que cuentan con una mejor regulación e información (3). El control por la vía de la elección de mercado se potencia cuando se desarrollan mecanismos complementarios de participación de la ciudadanía. 
Los procesos de reforma del sector de la salud constituyen una oportunidad para promover la participación y el control ciudadano sobre el sistema de salud, dado que se observa un bajo nivel de desarrollo de estos mecanismos en las distintas funciones del sistema. Generalmente, la incorporación de instrumentos de control ciudadano en el sector de la salud no formó parte del diseño original de las políticas. Sin embargo, las crecientes demandas y expectativas de la ciudadanía están ejerciendo presión sobre las autoridades para que se integre a los ciudadanos en los procesos de reforma en marcha. La escasa participación ciudadana se explica también por una actitud conservadora y de temor frente a los cambios por parte de los actores del sector y por un contexto que no ha favorecido el desarrollo de una ciudadanía más activa. Entre los principales obstáculos para el desarrollo de la participación en el sector de la salud se pueden mencionar los siguientes:

A. La desigualdad en la distribución de los ingresos y de otras formas de poder que caracteriza a los países de la Región. Esto se traduce en una baja cohesión e integración social que debilita la credibilidad de las instituciones (7).

B. El incipiente reconocimiento de los de rechos de las personas frente a los servicios públicos y la falta de instrumen tos para hacerlos efectivos. Ello se debe, en parte, a que la participación es vista aún como una concesión por parte de la autoridad, más que como un derecho inherente a todo ciudadano. Por otra parte, el concepto de participación en la salud se limita generalmente a la intervención de los actores internos del sistema, dejando fuera al resto de la sociedad (4).

C. La escasa información sobre los dere chos ciudadanos ante el sistema de sa lud y la ausencia de mecanismos que permitan hacerlos efectivos. Si bien existe vasta información en el sector de la salud, no está necesariamente disponible para los ciudadanos y en general no está orientada a facilitar el control del sistema por parte de los usuarios. Este escaso desarrollo de la información es, en parte, producto de una falta de organización de la ciudadanía y de los organismos de defensa de los consumidores. Sin embargo, es posible prever importantes cambios en esta mate- ria con el desarrollo de una ciudadanía con expectativas crecientes ante los servicios de salud (8).

D. La precariedad con que los mecanismos de control existentes reconocen la diversidad social y recogen la opinión de las minorías o de los sectores más mar ginados. Los países de América Latina y el Caribe se caracterizan por ser sociedades segmentadas, en las cuales un amplio porcentaje de la población cuenta con escasos recursos, poca información y poca capacidad de elección. Incorporar a estos sectores requiere superar la visión paternalista con la que tradicionalmente se plantea el sector de la salud frente a la población (8).

E. El débil desarrollo de las organizacio nes sociales que caracteriza a los países de América Latina y el Caribe. Por ello, en general, las políticas públicas, incluidas las políticas de participación, se construyen desde el Estado, más que por iniciativa de los ciudadanos. Muchas de las organizaciones existentes responden a políticas asistencialistas del Estado o a clientelismos $\mathrm{y}$, por ende, no representan la diversidad social o cuentan con poca legitimidad de sus representantes (8).

\section{REFERENCIAS}

1. Organización Panamericana de la Salud. Silos $\mathrm{N}^{\circ}$ 35. Desarrollo y fortalecimiento de los Sistemas Locales de Salud. En: La participación social en el desarrollo de la salud. Experiencias latinoamericanas. Washington, D.C.: OPS; 1995.

2. Celedón C, Noé M. Participación y gestión en salud. En: Hacia la implantación de modelos de administración gerencial en salud pública. XII Concurso de Ensayos del CLAD. Ensayos Premiados. Washington, D.C.: CLAD, OPS/OMS; 1999.

3. Londoño JL, Frenk J. Pluralismo estructurado: hacia un enfoque innovador para las reformas de los servicios de salud en América Latina. En:
Frenk J, ed. Observatorio de la Salud. México, D.F.: Fundación Mexicana para la Salud; 1997.

4. Sulbrandt J. Modernización del Estado y ciudadanía en los procesos de democratización. Una perspectiva desde América Latina. En: Dirección de Presupuestos, Ministerio de Hacienda. Calidad de Servicio y Atención al Usuario en el Sector Público. Santiago de Chile: Dolmen Ediciones; 1997

5. Sojo A. Hacia unas nuevas reglas del juego: los compromisos de gestión en salud de Costa Rica desde una perspectiva comparativa. Santiago de Chile: CEPAL; 1998.
6. Banco Interamericano de Desarrollo, Departamento Regional de Operaciones. Chile: antecedentes sobre el proceso de reforma del sector salud y escenarios posibles a futuro. Washington, D.C.: BID; 1999.

7. Kliksberg B, comp. Pobreza, un tema impostergable. Nuevas respuestas a nivel mundial. Caracas: Centro Latinoamericano de Administración para el Desarrollo, Fondo de Cultura Económica, Programa de Naciones Unidas para el Desarrollo; 1993.

8. Correa E, Noé M, eds. Nociones de una ciudadanía que crece. Santiago de Chile: FLACSO; 1998. 
ABSTRACT This article begins by briefly reviewing different forms of citizen participation in the health system. The article then suggests the need for mechanisms for society to control each of the tasks that the health system should perform: defining overall policies, financing, insurance, service provision, and evaluating the effectiveness and efficiency of the system. In general, in the countries of Latin America and the Caribbean, health sector policy formulation continues to be carried out in a centralized manner, with limited involvement by individuals and little adaptation to local realities. The reform processes in the Region are progressing in defining or improving the financing responsibility so as to better reconcile the objectives of equity, efficiency, and freedom of choice. Nevertheless, little has been done to develop instruments for citizens to control the functions of financing and insurance. Appropriate instruments are still lacking for citizens to effectively manage service providers. Among the principal obstacles to citizen participation in the health sector in the Region are: inequality in the distribution of income and other forms of power, an embryonic recognition of the rights of people when they are dealing with public services, limited information concerning those rights and the absence of mechanisms for people to truly exercise them, the weakness with which existing control mechanisms recognize social diversity and incorporate the views of minorities or of the most marginalized groups in society, and weak civic organizations. 